Núria Guañabens*, Xavier Filella, Ana Monegal, Carmen Gómez-Vaquero, María Bonet, Dolors Buquet, Enrique Casado, Dacia Cerdá, Alba Erra, Silvia Martinez, Núria Montalá, Concepción Pitarch, Eduardo Kanterewicz, Miquel Sala, Xavier Surís and Ferran Torres, on behalf of the LabOscat Study Group

\title{
Reference intervals for bone turnover markers in Spanish premenopausal women
}

DOI 10.1515/cclm-2015-0162

Received February 16, 2015; accepted May 18, 2015; previously published online June 18, 2015

\section{Abstract}

Background: The aims of this study were to establish robust reference intervals and to investigate the factors influencing bone turnover markers (BTMs) in healthy premenopausal Spanish women.

Methods: A total of 184 women (35-45 years) from 13 centers in Catalonia were analyzed. Blood and second void urine samples were collected between 8 a.m. and 10 a.m. after an overnight fast. Serum procollagen type I aminoterminal propeptide (PINP) and serum cross-linked C-terminal telopeptide of type I collagen (CTX-I) were measured by two automated assays (Roche and IDS), bone alkaline phosphatase (bone ALP) by ELISA, osteocalcin (OC) by IRMA and urinary NTX-I by ELISA. PTH and 25-hydroxyvitamin D (250HD) levels were measured. All participants completed a questionnaire on lifestyle factors.

Results: Reference intervals were: PINP: 22.7-63.1 and 21.8-65.5 $\mu \mathrm{g} / \mathrm{L}$, bone ALP: 6.0-13.6 $\mu \mathrm{g} / \mathrm{L}$, OC: $8.0-23.0$ $\mu \mathrm{g} / \mathrm{L}$, CTX-I: 137-484 and 109-544 ng/L and NTX-I:

*Corresponding author: Núria Guañabens, Service of Rheumatology, Hospital Clínic, IDIBAPS, CIBERehd, University of Barcelona, C/Villarroel 170, 08036 Barcelona, Spain, Phone: +34932275400 ext 2236, E-mail: nguanabens@ub.edu Xavier Filella: Biochemistry and Molecular Genetics Department, Hospital Clínic, Barcelona, Spain

Ana Monegal: Rheumatology Department, Hospital Clinic, IDIBAPS, CIBERehd, University of Barcelona, Barcelona, Spain

Carmen Gómez-Vaquero: Rheumatology Department, IDIBELL, Hospital Universitari de Bellvitge, L'Hospitalet, Spain

María Bonet: Rheumatology Department, Hospital de l'Alt Penedés, Villafranca del Penedés, Spain

Dolors Buquet: Rheumatology Department, Hospital Arnau de Vilanova, Lleida, Spain

Enrique Casado: Rheumatology Department, University Institute Parc Taulí, Sabadell, Spain

Dacia Cerdá: Rheumatology Department, Hospital Moisés Broggi, Barcelona, Spain
19.6-68.9 nM/mM. Oral contraceptive pills (OCPs) influenced PINP $(\mathrm{p}=0.007)$, and low body mass index (BMI) was associated with higher BTMs except for bone ALP. Women under 40 had higher median values of most BTMs. CTX-I was influenced by calcium intake $(\mathrm{p}=0.010)$ and PTH ( $p=0.007)$. 25OHD levels did not influence BTMs. Concordance between the two automated assays for PINP and particularly CTX-I was poor.

Conclusions: Robust reference intervals for BTMs in a Southern European country are provided. The effects of OCPs and BMI on their levels are significant, whilst serum 250HD levels did not influence BTMs. Age, calcium intake, BMI and PTH influenced CTX-I. The two automated assays for measuring PINP and CTX-I are not interchangeable.

Keywords: bone turnover markers; osteoporosis.

\section{Introduction}

Biochemical markers of bone turnover (BTMs) are used in the clinical setting for the initial assessment of osteoporosis, including the suspicion of secondary causes and the

Alba Erra: Rheumatology Department, Hospital San Rafael, Barcelona, Spain

Silvia Martinez: Rheumatology Department, Hospital Mútua de Terrassa, Terrassa, Spain

Núria Montalá: Rheumatology Department, Hospital Sta, María, Lleida, Spain

Concepción Pitarch: Rheumatology Department, Hospital Esperit Sant, Santa Coloma de Gramanet, Barcelona, Spain

Eduardo Kanterewicz: Rheumatology Department, Hospital de Vic, Vic, Spain

Miquel Sala: Rheumatology Department, Hospital de Figueres, Girona, Spain

Xavier Surís: Rheumatology Department, Hospital de Granollers, Granollers, Spain

Ferran Torres: Biostatistics and Data Management Core Facility, IDIBAPS, Hospital Clinic, Barcelona, Spain; and Biostatistics Unit, Faculty of Medicine, Universitat Autònoma de Barcelona, Barcelona, Spain 
identification of patients with rapid bone loss, but particularly for monitoring the response to treatment [1-3]. For these reasons, it is essential to have robust reference ranges of BTMs, and it is very important to have a reliable upper limit as well as a low limit value for each marker. High BTM levels, particularly those of bone resorption, have been linked with a high fracture risk, and if levels are increased greatly, causes of high bone turnover other than osteoporosis, such as hyperparathyroidism, myeloma or thyrotoxicosis, should be ruled out $[4,5]$. In addition, it has been considered that the goal of oral antiresorptive therapy was to reduce BTMs to within the lower half of the reference range interval for healthy premenopausal women [1]. With the post-marketing use of parenteral and more potent anti-resorptive drugs, such as zoledronic acid and denosumab [6,7], the lower limits of BTMs became more important than previously, as not only has it been questioned if decreases of BTMs below the reference interval could harm microdamage repair of the bone, but they also may be helpful in taking decisions in clinical practice, such as whether or not to give the next dose of a potent antiresorptive drug (e.g. zoledronic acid) [6-8].

At present, in some countries such as in Spain, reference intervals must be fine-tuned, since they were performed years ago on a low number of individuals [9], or were taken from the normalities of commercial brochures. In the last few years, reference intervals in premenopausal women from different countries in Europe (UK, France, Belgium and Denmark) [10, 11] and the US [11, 12] have been reported. In addition, data from Germany on men and women have been recently published [13]. In Spain, there are data on PINP and CTX-I in older men and in postmenopausal women [14-16], as well as on CTX-I in a subset of 50 premenopausal women [17]. As our aim was to establish reliable BTM intervals, it was tempting to use the reference intervals for BTMs in premenopausal women from a country with a geographical proximity, such as France [11]. However, we could not assume that the environmental and lifestyle factors are the same in French and in Spanish women. In fact, we were further interested in comparing our data with those reported in other European countries in order to evaluate if premenopausal women from a Southern European setting have different or similar bone remodeling activity than those from Western, Northern or Central Europe. Therefore, and taking into consideration the importance of the pre-analytical variability when establishing the reference intervals, we designed this study involving a large cohort of healthy women born in Spain, with an age range of between 35 and 45. This age range was taken because BTM levels were considered to be stable by Glover et al. in their study assessing the reference interval in premenopausal British women [18]. In addition, other sources of variability were minimized, since the circadian rhythm and the influence of feeding are well known contributing factors in the pre-analytical variability of BTMs [1]. Furthermore, the seasons of the recruitment period, and consequently vitamin D levels were taken into account as possible sources of variability, even in a sunny country, such as Spain.

The aims of this study were to establish robust reference intervals for BTMs in healthy premenopausal Spanish women and to analyze the factors influencing their levels.

\section{Materials and methods}

\section{Subjects}

A total of 185 women from 35 to 45 years of age (mean: 40.2+3.1) from 13 medical centers in Catalonia (Northeast of Spain) were recruited as volunteers. Most of them were health workers and their friends. The study was carried out between February 10 and June 13, 2013. All women were healthy, non-pregnant and were regularly menstruating. All participants completed a questionnaire on medical and lifestyle factors, including height and weight, previous pregnancies, alcohol consumption, tobacco habit (current smokers), dairy products intake, physical exercise, drugs, including oral contraceptives (OCP), and any medical condition. We calculated the body mass index (BMI) as the weight ( $\mathrm{kg}$ ) divided by height (m) squared; alcohol consumption as standard drink units (ethanol content of 8-10 g) per day; dietary calcium intake, by the number of portions of dairy products multiplied by the milligrams of calcium per portion per day, and physical activity according to four categories: from inactivity to high intensity exercise. We excluded women with a recent pregnancy or fracture (within 12 months) or with a surgical procedure in the last 3 months, as well as women with any medical condition interfering with bone metabolism. Women on OCP and those with low vitamin D serum levels were included in the study. Ethical approval from each participating center was obtained as well as written informed consent from each participant in the study.

\section{Biochemical and hormonal tests}

Blood and second void urine samples were collected between 8 and 10 a.m. after an overnight fast in all individuals. Serum and urine samples were stored at $-20{ }^{\circ} \mathrm{C}$ and plasma samples were stored at $-80{ }^{\circ} \mathrm{C}$ until analysis. All the assays were performed by a central laboratory.

Serum bone alkaline phosphatase (bone ALP) was measured by ELISA (Immunodiagnostic Systems, Boldon, UK), with an analytical sensitivity of $0.7 \mu \mathrm{g} / \mathrm{L}$. Serum cross-linked C-terminal telopeptide of type I collagen (CTX-I [Roche]) and total procollagen type I aminoterminal propeptide (PINP [Roche]) were measured using electrochemiluminescence automated immunoassays (Elecsys, Roche Diagnostics, Mannheim, Germany). The analytical sensitivities were $70 \mathrm{ng} / \mathrm{L}$ and $5 \mu \mathrm{g} / \mathrm{L}$, respectively. Serum CTX-I (CTX-I [IDS]) and intact 
Table 1: Demographic characteristics of all individuals. ${ }^{a}$

\begin{tabular}{lr}
\hline Age, years & $40(38-43)$ \\
BMI, kg/m² & $22.2(20.5-24.8)$ \\
Contraceptive pills & $20(10.9)$ \\
Previous fractures & $3(1.6)$ \\
Current smokers & $39(21.3)$ \\
Alcohol use & $40(21.9)$ \\
Alcohol intake units & $1(0.5-1)$ \\
Physical exercise & \\
$\quad$ Sporadic & $40(21.9)$ \\
$\quad$ Regular, low intensity & $77(42.1)$ \\
$\quad$ Regular, high intensity & $25(13.7)$ \\
Previous pregnancy & $133(73.5)$ \\
Previous nephrolithiasis & $16(8.7)$ \\
Calcium intake, mg/d & $621.1(479.1-869.5)$ \\
25-hydroxyvitamin D, nmol/L & $44.93(32.4-54.9)$ \\
PTH, pmol/L & $4.5(3.4-6.0)$ \\
\hline
\end{tabular}

Statistics are $\mathrm{n}$ (\%) or median (25th-75th percentiles). alncluding oral contraceptive users.

PINP (PINP [IDS]) were also measured using chemiluminescence automated immunoassays (IDS-ISYS, Immunodiagnostic Systems). The analytical sensitivities were $33 \mathrm{ng} / \mathrm{L}$ and $1 \mu \mathrm{g} / \mathrm{L}$, respectively. Urinary cross-linked N-terminal telopeptide of type I collagen (NTX-I) was measured by ELISA (Osteomark ${ }^{\circledR}$ NTX-I, Alere, Scarborough, ME, USA), with an analytical sensitivity of $20 \mathrm{nM}$. NTX-I was expressed as a ratio to creatinine. Osteocalcin (OC) was measured by IRMA (Cis Bio, Sorgues, France), with an analytical sensitivity of $0.4 \mu \mathrm{g} / \mathrm{L}$.

Plasma parathyroid hormone (PTH) (Centaur XP, Siemens) and serum 25-hydroxyvitamin D (250HD) (Liaison, Diasorin, Stillwater, MN, USA) were measured using automated immunoassays in all participants. The analytical sensitivities were $0.3 \mathrm{pmol} / \mathrm{L}$ and 9.98 $\mathrm{nmol} / \mathrm{L}$, respectively. Reference ranges were 1.1-6.8 pmol/L for PTH. A concentration of $25 \mathrm{OHD}<50 \mathrm{nmol} / \mathrm{L}$ was considered as vitamin D deficiency.

\section{Statistical analysis}

A quantile regression was used to estimate the 5\%, 50\% and 95\% percentiles and their $95 \% \mathrm{CI}$ for the BMTs and to obtain unadjusted and OCP adjusted p-values. Standard non-parametric tests for continuous variables and the Fisher's exact test were used to assess the influence of factors on BTMs. To compare the two analytic assays we used the Bland-Altman method as well as the Lin's concordance coefficient (LCC) and the corresponding 95\% CI. The Bland-Altman $95 \%$ limits of agreement (mean bias $\pm 2 \mathrm{SD}$ ) procedure, based on comparison of the mean of two methods against its difference, uses datascale assessment to analyze the accuracy (i.e. bias) and the amount of variation or precision between any two measured values $[19,20]$. The Lin coefficient combines measures of both precision and accuracy to determine whether the observed data deviate significantly from the line of perfect concordance, which occurs at $45^{\circ}$, i.e. it assesses the linear relationship between two variables under the constraint where the intercept is zero and the slope is one. The value of Lin's coefficient increases as the accuracy and precision of the observed data improve [21-23].

\section{Results}

In this study, 185 premenopausal women were recruited, and of these women we analyzed data from 184 (one subject was excluded because of unreliable outlier values in all BTMs). Their demographic characteristics are shown in Table 1. Reference intervals for all BTMs (5\%, 50\% and 95\% percentiles, as well as their 95\% CI) obtained from the entire group of women and from the 164 women who were not on OCP are shown in Table 2. Thus, OCP in 20 women $(10.9 \%)$ influenced PINP levels $(\mathrm{p}=0.007)$ with a trend on bone ALP levels $(\mathrm{p}=0.09)$.

Age (cut-off point at 40 years), when adjusted by oral contraceptive use, was not significant for any of the bone marker values (Bone ALP $\mathrm{p}=0.273$, PINP [Roche] $\mathrm{p}=0.272$, PINP [IDS] $\mathrm{p}=0.288$, CTX-I-Roche $\mathrm{p}=0.306$, CTX-I [IDS] $\mathrm{p}=0.318$, NTX-I $\mathrm{p}=0.253$, OC $\mathrm{p}=0.215$ ). However, when excluding OCP users, there were significant differences or trends for all BTM values when women from 35 to 39 and 40 to 45 were analyzed separately. Thus, all median values were higher in women under 40 , although the observed relative differences were $<15 \%$ of the normal reference intervals.

Table 2: Reference values for the bone turnover markers in all individuals and in non-oral contraceptive users.

\begin{tabular}{|c|c|c|c|c|c|c|}
\hline & \multicolumn{3}{|c|}{ All individuals $(n=184)$} & \multicolumn{3}{|c|}{ Non-oral contraceptive users $(n=164)$} \\
\hline & $\begin{array}{r}\text { Percentile } 5 \\
(95 \% \mathrm{Cl})\end{array}$ & $\begin{array}{r}\text { Percentile } 50 \\
(95 \% \mathrm{Cl})\end{array}$ & $\begin{array}{r}\text { Percentile } 95 \\
(95 \% \mathrm{Cl})\end{array}$ & $\begin{array}{r}\text { Percentile } 5 \\
(95 \% \mathrm{Cl})\end{array}$ & $\begin{array}{r}\text { Percentile } 50 \\
(95 \% \mathrm{Cl})\end{array}$ & $\begin{array}{r}\text { Percentile } 95 \\
(95 \% \mathrm{CI})\end{array}$ \\
\hline Bone ALP, $\mu \mathrm{g} / \mathrm{L}$ & $6.0(5.4-6.6)$ & $9.3(8.8-9.8)$ & $13.8(12.0-15.5)$ & $6.0(5.3-6.7)$ & $9.4(8.9-9.9)$ & $13.6(11.8-15.4)$ \\
\hline PINP (Roche), $\mu \mathrm{g} / \mathrm{L}$ & $20.8(18.4-23.2)$ & $35.9(33.8-37.9)$ & $60.6(52.0-69.1)$ & $22.7(20.0-25.5)$ & $36.0(33.8-38.2)$ & $63.1(51.8-74.4)$ \\
\hline PINP (IDS), $\mu \mathrm{g} / \mathrm{L}$ & $20.8(18.9-22.7)$ & $35.8(33.2-38.3)$ & $64.9(57.1-72.7)$ & $21.8(19.6-24.0)$ & $36.6(34.0-39.1)$ & $65.5(54.0-77.0)$ \\
\hline NTX-I, nM/mM & $19.3(15.9-22.7)$ & $32.7(30.1-35.3)$ & $68.9(60.1-77.8)$ & $19.6(15.9-23.3)$ & $32.9(29.7-36.1)$ & $68.9(55.8-82.1)$ \\
\hline CTX-I (Roche), ng/L & $137(122-152)$ & $250(230-270)$ & $480(429-531)$ & $137(120-154)$ & $255(234-276)$ & $484(351-617)$ \\
\hline CTX-I (IDS), ng/L & $107(92-123)$ & $246(219-272)$ & $541(439-643)$ & $109(91-127)$ & $249(222-277)$ & $544(408-680)$ \\
\hline $\mathrm{OC}, \mu \mathrm{g} / \mathrm{L}$ & $8.0(6.7-9.3)$ & $14.0(13.4-14.6)$ & $23.0(20.4-25.6)$ & $8.0(7.1-8.9)$ & $14.0(13.3-14.7)$ & $23.0(19.9-26.1)$ \\
\hline
\end{tabular}




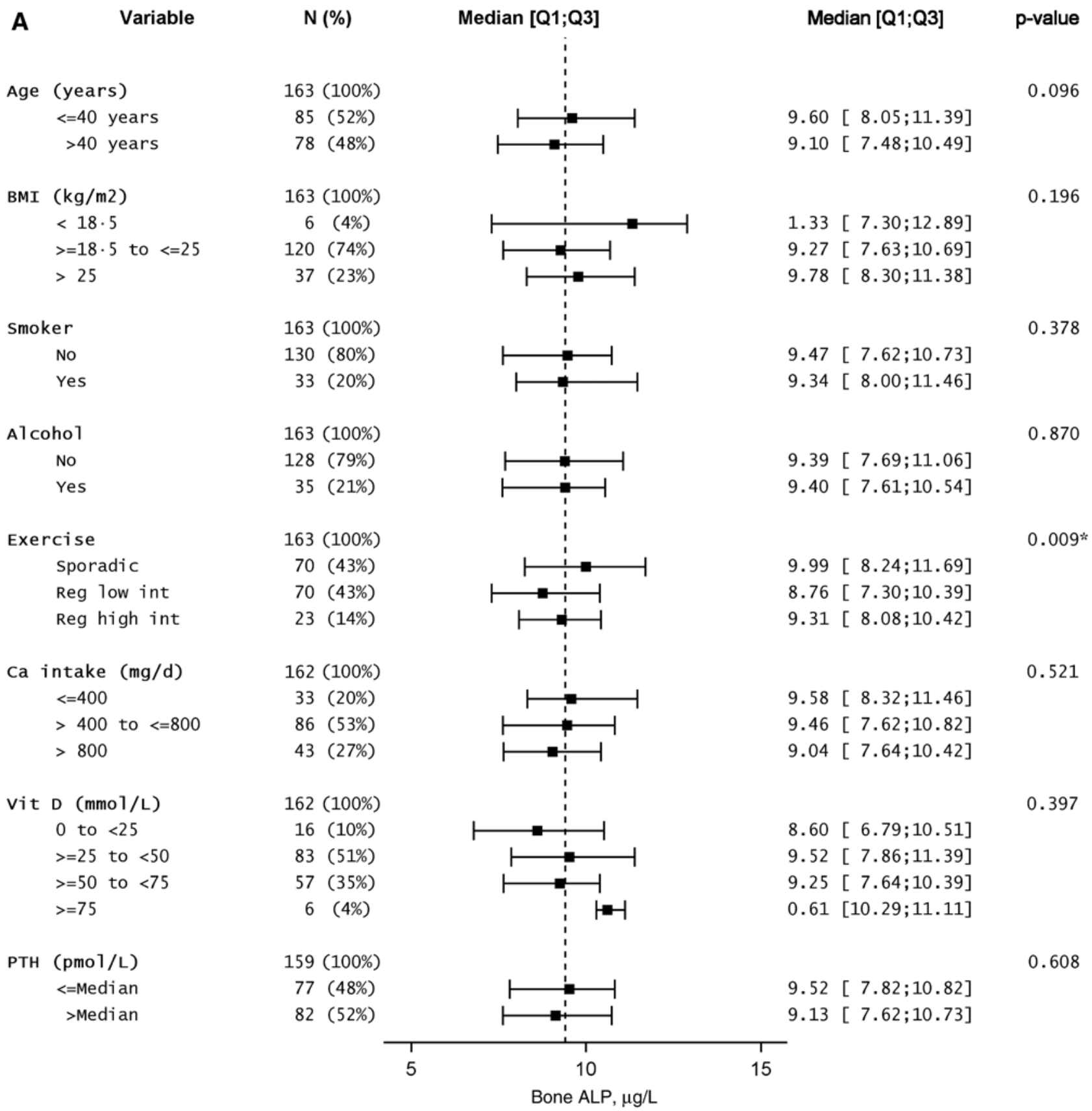

Figure 1 (continued)

After excluding OCP users, low BMI was associated with higher levels of all BTMs except for bone ALP, when comparing women according to their median value of BMI. By contrast, neither alcohol consumption, observed in $21.9 \%$ of subjects in low amounts (1 [0.5-1] units), nor current smoking (20.2\% of subjects) showed any effect on BTM levels. Exercise-influenced bone ALP, but not other BTM levels when subjects were classified according to those engaged in regular exercise and those who were sedentary (8.9 [7.5-10.4] vs. 9.9 [8.2-11.7] $\mu \mathrm{g} / \mathrm{L}, \mathrm{p}=0.005)$.
Dietary calcium intake influenced CTX-I [Roche] and CTX-I [IDS] serum levels, as CTX-I [Roche] decreased as the calcium intake increased $(\leq 400,>400-\leq 800$ and $>800$ $\mathrm{mg} /$ day): 290 (210-400), 260 (190-320) and 220 (170-280) $\mathrm{ng} / \mathrm{L}$, respectively, $\mathrm{p}=0.010$; CTX-I [IDS] levels showed the same trend $(p=0.024)$. The median value of $250 \mathrm{HD}$ was $44.9(32.4,54.9) \mathrm{nmol} / \mathrm{L}$, with levels of $25 \mathrm{OHD}$ being higher than $50 \mathrm{nmol} / \mathrm{L}$ in nearly $40 \%$ of subjects. There were no differences in any BTM levels when comparing women with those above or below the median value or according 


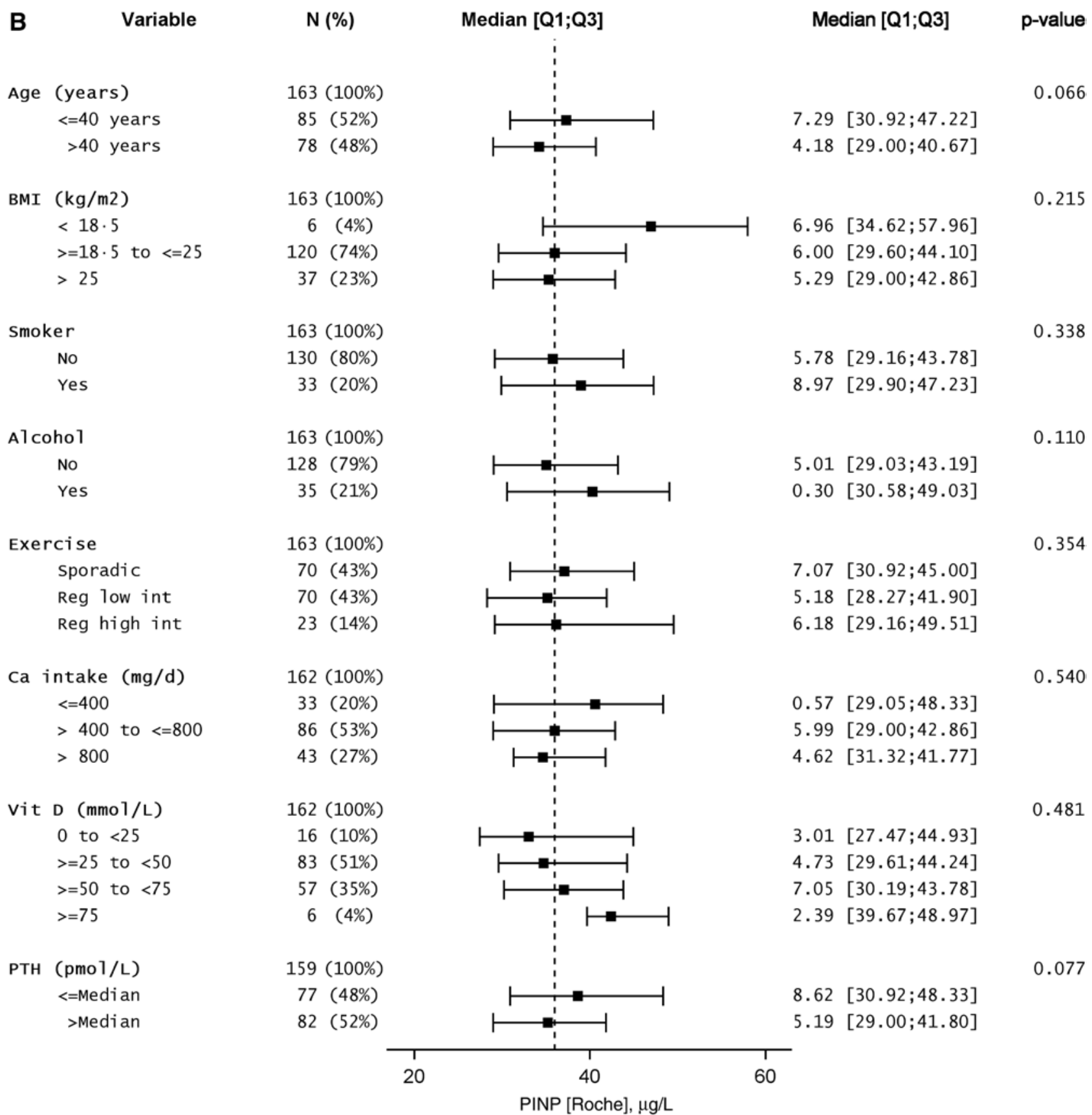

Figure 1 (continued)

to 25OHD tertiles (p33: 34.94; p66: $49.92 \mathrm{nmol} / \mathrm{L}$ ). However, PTH showed a statistically significant relationship with CTX-I [Roche] when assessed both as a continuous variable $(p=0.007)$ and when using the median as a cut-off point $(\mathrm{p}=0.016)$. By contrast, CTX-I [IDS] was not associated with PTH levels ( $\mathrm{p}=0.305$ and $\mathrm{p}=0.312$, respectively). Figure 1 shows the influence of different variables according to the median and Q1; Q3 values.

The Lin's concordance (95\% CI) between the two automated assays used for measuring CTX-I and PINP was
0.851 (0.812-0.883) for CTX-I and 0.878 (0.841-0.906) for PINP. The Bland-Altman bias (95\% limits of agreement) was $-0.011(-0.153-0.131)$ and $-1.282(-14.026-11.461)$ for CTX-I and PINP, respectively (Figure 2, Table 3).

\section{Discussion}

This study provides robust reference intervals for BTMs in Spanish women and contributes to the identification 


\begin{tabular}{|c|c|c|}
\hline C & Variable & $\mathrm{N}(\%)$ \\
\hline Age & (years) & $149(100 \%)$ \\
\hline & $<=40$ years & $75(50 \%)$ \\
\hline & $>40$ years & $74(50 \%)$ \\
\hline BMI & $(\mathrm{kg} / \mathrm{m} 2)$ & $149(100 \%)$ \\
\hline & $<18 \cdot 5$ & $6(4 \%)$ \\
\hline & $>=18 \cdot 5$ to $<=25$ & $110(74 \%)$ \\
\hline & $>25$ & $33(22 \%)$ \\
\hline Smok & ker & $149(100 \%)$ \\
\hline & No & 117 (79\%) \\
\hline & Yes & $32(21 \%)$ \\
\hline Al Co & ohol & $149(100 \%)$ \\
\hline & No & $114(77 \%)$ \\
\hline & Yes & $35(23 \%)$ \\
\hline Exer & rcise & $149(100 \%)$ \\
\hline & Sporadic & $61(41 \%)$ \\
\hline & Reg low int & $66(44 \%)$ \\
\hline & Reg high int & $22(15 \%)$ \\
\hline & intake $(\mathrm{mg} / \mathrm{d})$ & $148(100 \%)$ \\
\hline & $<=400$ & $32(22 \%)$ \\
\hline & $>400$ to $<=800$ & $76(51 \%)$ \\
\hline & $>800$ & $40(27 \%)$ \\
\hline vit & $\mathrm{D}(\mathrm{mmol} / \mathrm{L})$ & $150(100 \%)$ \\
\hline & 0 to $<25$ & $12(8 \%)$ \\
\hline & $>=25$ to $<50$ & $76(51 \%)$ \\
\hline & $>=50$ to $<75$ & $56(37 \%)$ \\
\hline & $>=75$ & $6(4 \%)$ \\
\hline PTH & $(\mathrm{pmol} / \mathrm{L})$ & $145(100 \%)$ \\
\hline & $<=$ Median & $69(48 \%)$ \\
\hline & $>$ Median & $76(52 \%)$ \\
\hline
\end{tabular}

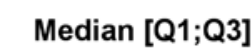

Median [Q1;Q3]

p-value

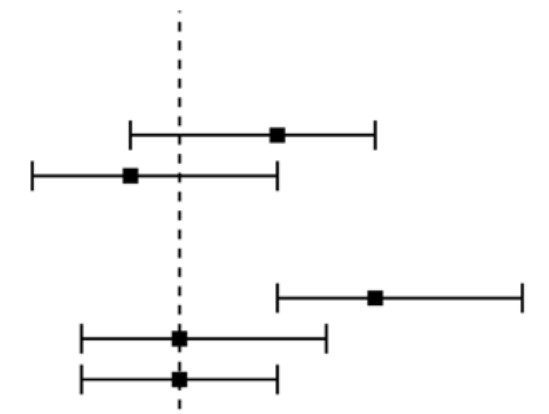

$6.00[13.00 ; 18.00]$

$3.00[11.00 ; 16.00]$

$8.00[16.00 ; 21.00]$

$4.00[12.00 ; 17.00]$

$4.00[12.00 ; 16.00]$

0.884

$4.00[12.00 ; 16.70]$

$4.00[11.00 ; 19.00]$

0.610

$4.00[12.00 ; 17.00]$

$4.00[12.00 ; 18.00]$

0.605

$5.00 \quad[12.00 ; 16.70]$

$4.00 \quad[12.00 ; 17.00]$

$3.50[12.00 ; 18.00]$

0.149

$6.00[12.00 ; 18.00]$

$4.00[12.00 ; 18.00]$

$3.00[12.00 ; 16.00]$

$0.046^{*}$

$3.50[10.90 ; 17.50]$

$4.00[12.00 ; 16.00]$

$4.00[12.00 ; 17.50]$

$8.50[18.00 ; 21.00]$

0.493
$4.00[12.00 ; 18.00]$

$4.00[12.50 ; 16.85]$
$0.004 \%$

0.052

.

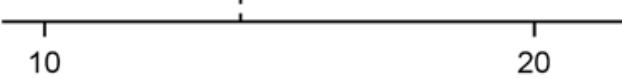

$\mathrm{OC}, \mu \mathrm{g} / \mathrm{L}$

Figure 1 (continued)

of reference ranges for BTMs in Southern Europe. In addition, our results reinforce the effects of OCP and BMI on their levels, as OCP use was associated with lower levels of most bone formation markers and low BMI-induced higher levels of most BTMs. Interestingly, calcium intake influences serum CTX-I values.

It is of value to assess whether reference intervals for BTMs in premenopausal women differ according to the location of the country in Europe. This has been analyzed by Glover et al., comparing European data from UK,
France and Belgium [11]. Surprisingly, Spanish values for bone ALP, PINP and serum CTX-I were quite similar to UKreported values, instead of those of French women from our neighboring country. Indeed, PINP and CTX-I, higher in France than in UK have been attributed to different lifestyle factors and BMI [11]. In addition, we found similar PINP and serum CTX-I values to those published by other authors in Italian and in German premenopausal women aged 35-45 years, particularly for total and intact PINP $[13,24]$. However, these affirmations must be taken with 


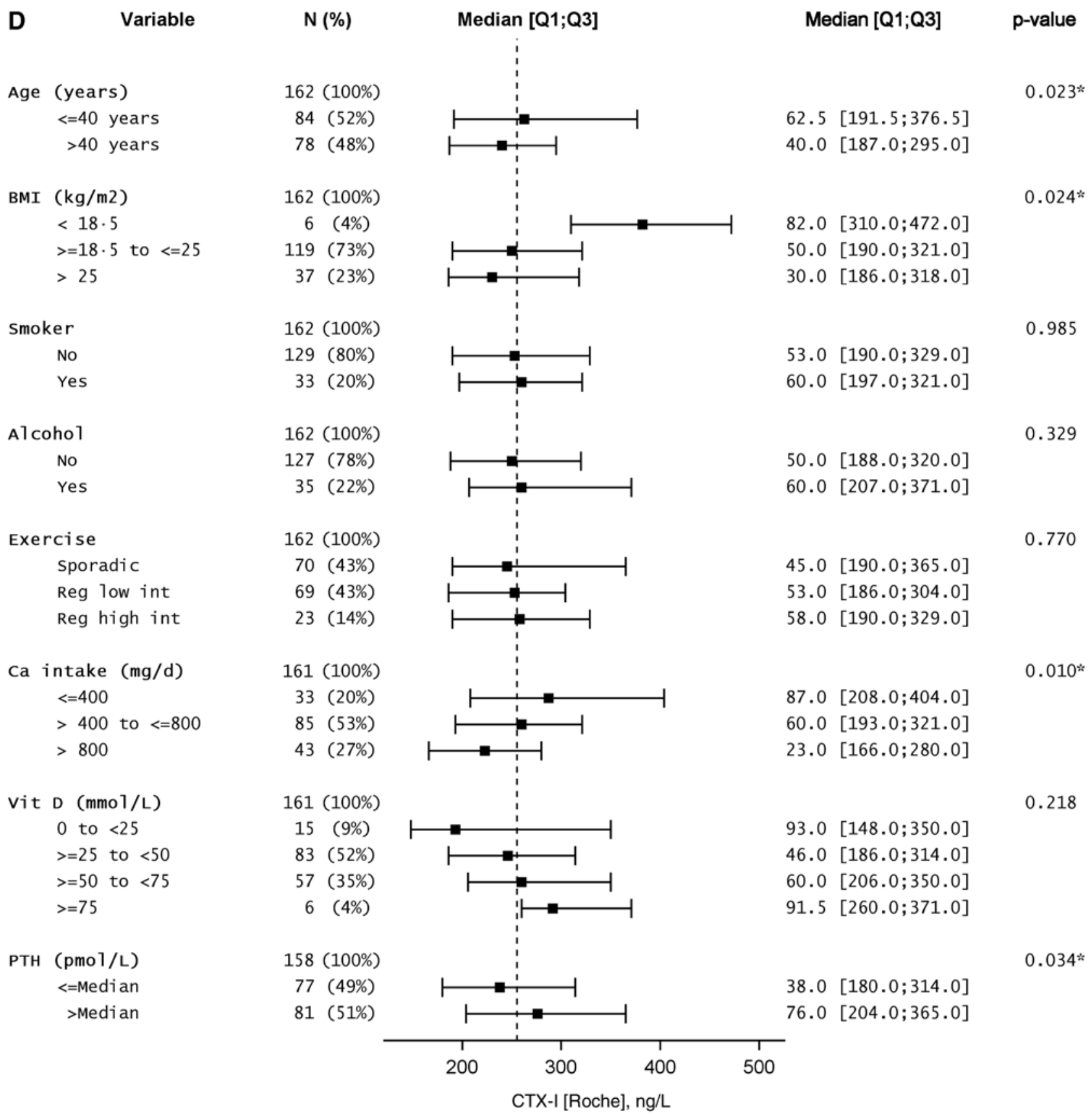

Figure 1 (continued)

caution as the compared studies were performed not only in different populations, but also under different analytical conditions. Thus, although all of them reported reference intervals for PINP and serum CTX-I using automated assays, samples were analyzed in different batches, and some of them in different laboratories. In addition, there were some discordant pre-analytical conditions. Most studies were performed in premenopausal women but the age ranges were not identical, and contrary to Glover et al. [18], we have found that women from 35 to 40 have slightly higher BTM values ( $<15 \%$ in magnitude) than those between 40 and 45 years of age. In addition, most, but not all samples were collected between 8 a.m. and 10 a.m. Interestingly, Australian harmonized reference intervals for PINP and CTX-I in premenopausal women have been recently published, and the intervals are much wider. Indeed, the reference intervals for PINP [Roche] are $15-70 \mathrm{ng} / \mathrm{mL}$ in an age range of $25-49$ years, with values of $15-90 \mathrm{ng} / \mathrm{mL}$ in women aged $20-24$ years [25]. These results suggest that the reference intervals for PINP as well 


\begin{tabular}{|c|c|c|}
\hline E & Variable & $\mathrm{N}(\%)$ \\
\hline \multirow[t]{3}{*}{ Age } & (years) & $161(100 \%)$ \\
\hline & $<=40$ years & $85(53 \%)$ \\
\hline & $>40$ years & $76(47 \%)$ \\
\hline \multirow[t]{4}{*}{ BMI } & $(\mathrm{kg} / \mathrm{m} 2)$ & $161(100 \%)$ \\
\hline & $<18.5$ & $6(4 \%)$ \\
\hline & $>=18 \cdot 5$ to $<=25$ & 119 (74\%) \\
\hline & $>25$ & $36(22 \%)$ \\
\hline \multicolumn{2}{|c|}{ Smoker } & $161(100 \%)$ \\
\hline & No & $130(81 \%)$ \\
\hline & Yes & 31 (19\%) \\
\hline \multicolumn{2}{|c|}{ Alcohol } & $161(100 \%)$ \\
\hline & No & 127 (79\%) \\
\hline & Yes & $34(21 \%)$ \\
\hline \multicolumn{2}{|c|}{ Exercise } & $161(100 \%)$ \\
\hline & Sporadic & $68(42 \%)$ \\
\hline & Reg low int & $70(43 \%)$ \\
\hline & Reg high int & $23(14 \%)$ \\
\hline \multirow{2}{*}{\multicolumn{2}{|c|}{$\begin{array}{c}\text { Ca intake }(\mathrm{mg} / \mathrm{d}) \\
<=400\end{array}$}} & $160(100 \%)$ \\
\hline & & 32 (20\%) \\
\hline & $>400$ to $<=800$ & $85(53 \%)$ \\
\hline & $>800$ & $43(27 \%)$ \\
\hline \multirow[t]{5}{*}{ vit } & $\mathrm{D}(\mathrm{mmol} / \mathrm{L})$ & $160(100 \%)$ \\
\hline & 0 to $<25$ & $16(10 \%)$ \\
\hline & $>=25$ to $<50$ & $81(51 \%)$ \\
\hline & $>=50$ to $<75$ & $57(36 \%)$ \\
\hline & $>=75$ & $6(4 \%)$ \\
\hline \multirow[t]{3}{*}{ PTH } & $(\mathrm{pmol} / \mathrm{L})$ & 157 (100\%) \\
\hline & $<=$ Median & $75(48 \%)$ \\
\hline & $>$ Median & $82(52 \%)$ \\
\hline
\end{tabular}

Median [Q1;Q3]

Median [Q1;Q3]

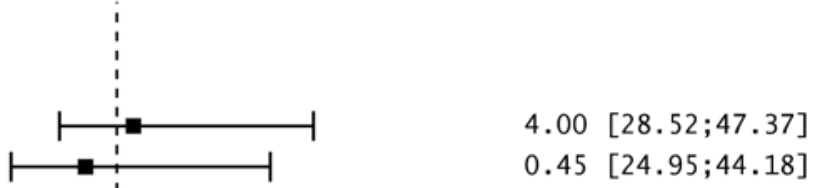

$0.45[24.95 ; 44.18]$

$9.15[35.74 ; 55.53]$

$1.98[26.41 ; 45.21]$

$3.87[24.94 ; 44.34]$

0.342

$1.92[26.16 ; 45.21]$

$9.05[26.14 ; 46.31]$

0.204

$1.87[26.00 ; 45.21]$

$7.24[26.61 ; 48.07]$

0.810

$2.00 \quad[26.07 ; 44.92]$

$5.33[26.41 ; 46.06]$

$1.29[26.16 ; 49.84]$

0.072

$9.61[28.10 ; 53.44]$

$1.53[26.16 ; 44.32]$

$0.50[25.37 ; 44.21]$

0.416

$1.38[25.03 ; 40.60]$

$2.94[27.00 ; 45.48]$

$1.87[25.37 ; 46.06]$

$8.51[30.74 ; 49.84]$

0.880

0.119

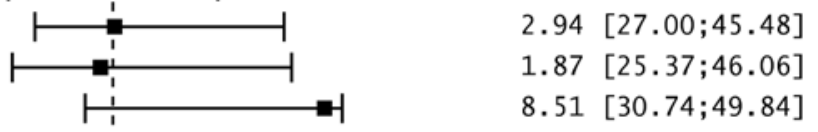

p-value

$0.038^{*}$

(1)

$3.39[25.72 ; 47.98]$

$2.56[27.00 ; 45.18]$

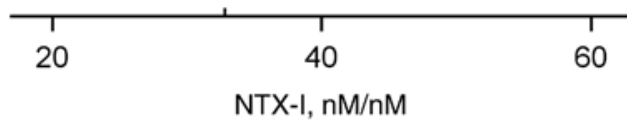

9



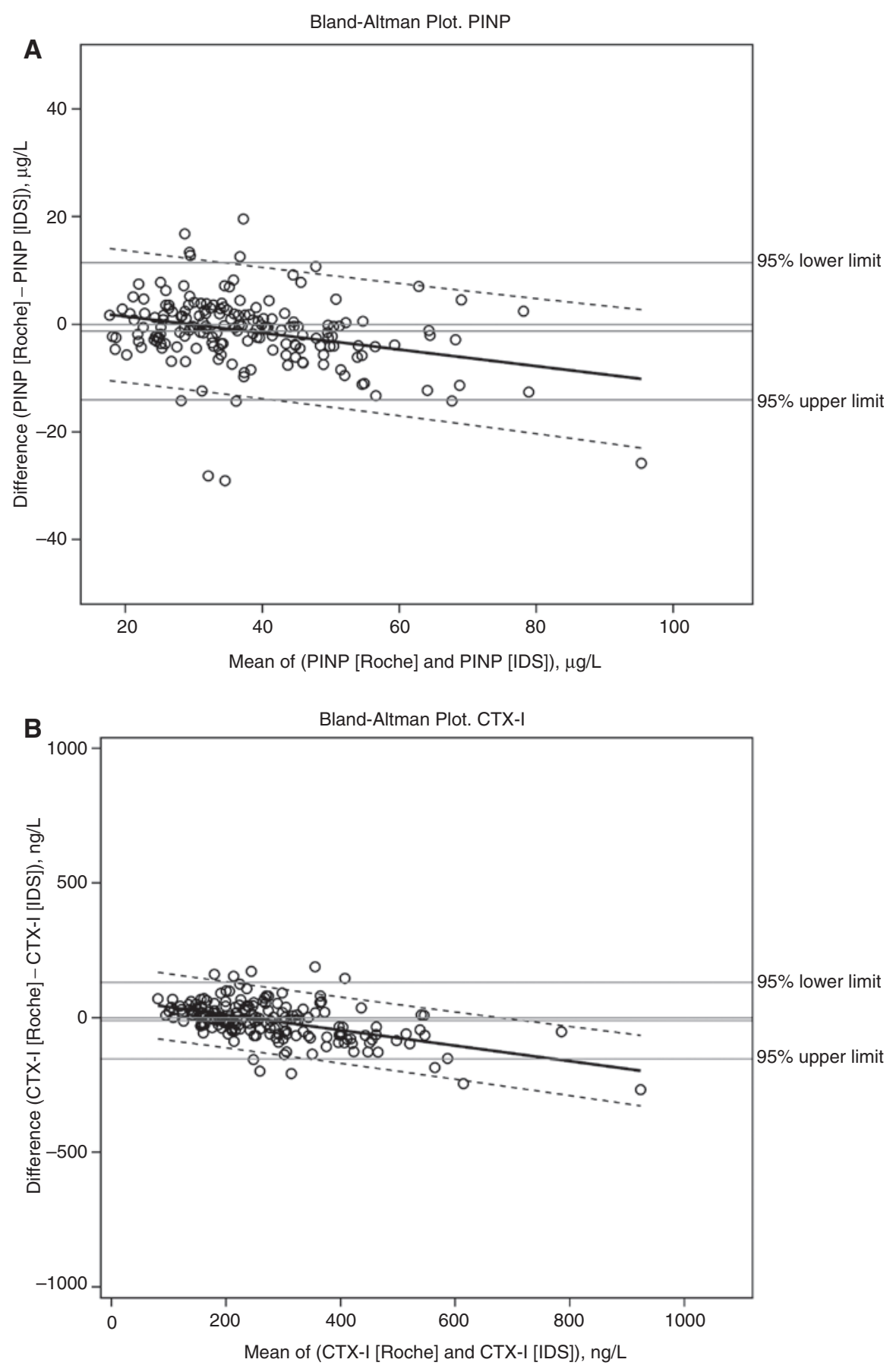

Figure 2: Bland-Altman plots of agreement.

(A) PINP; (B) CTX-I. CTX-I [IDS], IDS-ISYS serum cross-linked C-terminal telopeptide of type I collagen; CTX-I [Roche], Elecsys serum crosslinked C-terminal telopeptide of type I collagen; PINP [IDS], intact procollagen type I amino-terminal propeptide; PINP [Roche], total procollagen type I amino-terminal propeptide.

levels did not affect CTX-I values, and bone ALP was the only BTM correlated with PTH [10]. Similar to our results, Michelsen et al. found an association between PTH and CTX-I [13].

The relationship between BTMs and 25OHD levels has received particular attention in most recent publications.
We found no difference in BTM levels when classifying individuals according to the median values or to tertiles, or according to levels higher or $<50 \mathrm{nmol} / \mathrm{L}$ of $250 \mathrm{HD}$. Only OC showed different values in vitamin D-replete women when compared with those with vitamin D insufficiency or deficiency ( $25 \mathrm{OHD}<75$ and $<50 \mathrm{nmol} / \mathrm{L}$, respectively). 
Table 3: Bland and Altman Bias and Lin's concordance coefficient for the two assays.

\begin{tabular}{|c|c|c|}
\hline & CTX-I & PINP \\
\hline \multicolumn{3}{|l|}{ Bland-Altman agreement ${ }^{\mathrm{a}}$} \\
\hline Bias $(95 \% \text { limits of agreement })^{b}$ & $-11.215(-153.099-130.668)$ & $-1.282(-14.025-11.461)$ \\
\hline Intercept $(95 \% \mathrm{CI})^{\mathrm{c}}$ & $67.994(46.273-89.715)$ & $4.513(1.707-7.319)$ \\
\hline Slope $(95 \% \mathrm{Cl})^{c}$ & $-0.286(-0.357$ to -0.215$)$ & $-0.154(-0.224$ to -0.083$)$ \\
\hline Lin’s concordance coefficient $(95 \% \mathrm{Cl})$ & $0.851(0.812-0.883)$ & $0.878(0.841-0.906)$ \\
\hline \multirow[t]{2}{*}{ Pearson lineal correlation coefficient (Ro) ( $p$-value) } & 0.886 & 0.891 \\
\hline & $(p<0.001)$ & $(p<0.001)$ \\
\hline
\end{tabular}

$\mathrm{Cl}$, confidence intervals; SD, standard deviation. ${ }^{\mathrm{a} A v e r a g e ~ v e r s u s ~ m e a n ~ d i f f e r e n c e ~ a s s e s s m e n t ~ f o r ~ t h e ~ p a i r w i s e ~ c o m p a r i s o n s ; ~}{ }^{\mathrm{b}} \mathrm{Mean}$ bias; 'Slope and intercept for the average versus mean difference assessment linear regression.

However, the low number of vitamin-D replete individuals $(n=6)$ makes these differences of doubtful significance. Also, some authors have not found significant differences in BTMs when premenopausal women were classified in vitamin D deficient, insufficient or replete categories [18]. Yet other studies found a significant correlation between 25OHD levels and bone ALP [10, 11, 13]. It may be argued that 250HD levels in our individuals were low, as the median value was $44.9 \mathrm{nmol} / \mathrm{L}$, and more than $50 \%$ of them had 250HD levels $<50 \mathrm{nmol} / \mathrm{L}$. Nonetheless, these are the values in healthy young women from our sunny country between February and June.

A relevant contribution of this study is the comparison of the two major automated assays used in Europe to perform PINP and CTX-I measurements. We found that there was a poor concordance and a clear trend with a negative slope for both analytes, and therefore, the interpretation of the bias could not be considered homogeneous along the observed values of CTX-I and PINP. The mean differences were more different from the null (zero differences) for larger values. Although the results were not homogeneous, median levels of PINP [Roche] were very similar to the median levels of intact PINP (PINP [IDS]) ( 36.0 and $36.6 \mu \mathrm{g} / \mathrm{L}$, respectively), and we found higher median values for Elecsys CTX-I (CTX-I [Roche]) when compared with the IDS-ISYS assay (CTX-I [IDS]) (255 and $249 \mathrm{ng} / \mathrm{L}$, respectively). Also, it has been pointed out that the major automated assays for measuring PINP provide harmonized results [28]. Although the reference intervals are similar, particularly when measuring PINP, our results support the fact that the obtained values are not interchangeable. As a consequence, the interpretation of PINP and CTX-I results and their reference intervals need to be established for each assay method, most importantly for CTX-I.

The strengths of this study are the homogeneous preanalytical and analytical conditions, such as a narrow age range of healthy women between 35 and 45 years of age, morning fasting samples between 8:00 and 10:00 a.m. and a central specialized laboratory, which decrease the degree of variability. The limitations of this study are the low number of individuals, the absence of bone mineral density data and the low values of $250 \mathrm{HD}$ in a large proportion of the series. However, this fact reflects the vitamin D status in healthy young women in our country.

In conclusion, this study provides reference intervals of BTMs in healthy young women from a sunny Southern European country. Interestingly, BTM values are similar to those reported in UK, Germany and Italy, but different from Belgium and France, our neighbouring country. Taken together, it is recommended to establish reference intervals in each country, as a geographical neighbourhood does not imply exchangeable values for BTMs. Furthermore, reference intervals must be based on measurements with the same assay.

Acknowledgments: This study was funded by a research grant from the Catalan Society of Rheumatology. The kits were generously provided by Roche Diagnostics and by Immunodiagnostic Systems, Spain.

Author contributions: All the authors have accepted responsibility for the entire content of this submitted manuscript and approved submission.

Research funding: None declared.

Employment or leadership: None declared.

Honorarium: None declared.

Competing interests: The funding organization(s) played no role in the study design; in the collection, analysis, and interpretation of data; in the writing of the report; or in the decision to submit the report for publication.

\section{LabOscat Study group}

Marta Larrosa, Rheumatology Department, University Institute Parc Taulí, Sabadell; Joan Miquel Nolla, Rheumatology Department, IDIBELL, Hospital Universitari de 
Bellvitge, L'Hospitalet, Barcelona; Pilar Peris, Rheumatology Department, Hospital Clinic, IDIBAPS, CIBERehd, Barcelona; Daniel Roig-Vilaseca, Rheumatology Department, Hospital Moisés Broggi, Barcelona.

\section{References}

1. Naylor K, Eastell R. Bone turnover markers: use in osteoporosis. Nat Rev Rheumatol 2012;8:379-89.

2. Biver E, Chopin F, Coiffier G, Brentano TF, Bouvard B, Garnero P, et al. Bone turnover markers for osteoporotic status assessment? A systematic review of their diagnosis value at baseline in osteoporosis. J Bone Spine 2012;79:20-5.

3. Garnero P. Bone markers in osteoporosis. Curr Osteoporos Rep 2009;7:84-90.

4. Cerdá Gabaroi D, Peris P, Monegal A, Albaladejo C, Martínez MA, Muxí A, et al. Search for hidden secondary causes in postmenopausal women with osteoporosis. Menopause 2010;17:135-9.

5. Fitzpatrick L. Secondary causes of osteoporosis. Mayo Clin Proc 2002;77:453-68.

6. Grey A, Bolland M, Wattie D, Horne A, Gamble G, Reid IR. Prolonged antiresorptive activity of zoledronate: a randomized, controlled trial. J Bone Miner Res 2010;25:2251-5.

7. Eastell R, Christiansen C, Grauer A, Kutilek S, Libanati C, McClung MR, et al. Effects of denosumab on bone turnover markers in postmenopausal osteoporosis. J Bone Miner Res 2011;26:530-7.

8. Reid IR, Black DM, Eastell R, Bucci-Rechtweg C, Su G, Hue TF, et al. HORIZON Pivotal Fracture Trial and HORIZON Recurrent Fracture Trial Steering Committees. Reduction in the risk of clinical fractures after a single dose of zoledronic acid 5 milligrams. J Clin Endocrinol Metab 2013;98:557-63.

9. Peris P, Alvarez L, Monegal A, Guañabens N, Durán M, Pons F, et al. Biochemical markers of bone turnover after surgical menopause and hormone replacement therapy. Bone 1999;25:349-53.

10. Eastell R, Garnero P, Audebert C, Cahall DL. Reference intervals of bone turnover markers in healthy premenopausal women: results from a cross-sectional European study. Bone 2012;50:1141-7.

11. Glover SJ, Gall M, Schoenborn-Kellenberger O, Wagener M, Garnero P, Boonen S, et al. Establishing a reference interval for bone turnover markers in 637 healthy, young, premenopausal women from the United Kingdom, France, Belgium and the United States. J Bone Miner Res 2009;24:389-97.

12. de Papp AE, Bone HG, Caulfield MP, Kagan R, Buinewicz A, Chen $E$, et al. A cross-sectional study of bone turnover markers in healthy premenopausal women. Bone 2007;40:1222-30.

13. Michelsen J, Wallaschofski H, Friedrich N, Spielhagen C, Rettig R, Ittermann T, et al. Reference intervals for serum concentrations of three bone turnover markers for men and women. Bone 2013;57:399-404.
14. Martínez J, Olmos JM, Hernández JL, Pinedo G, Llorca J, Obregón E, et al. Bone turnover markers in Spanish postmenopausal women: the Camargo cohort study. Clin Chim Acta 2009;409:70-4.

15. Kanterewicz E, Peris P, Puigoriol E, Yáñez A, Rosique P, Del Rio L; FRODOS Research Group. Distribution of serum $\beta C T X$ in a population-based study of postmenopausal women taking into account different anti-osteoporotic therapies (the FRODOS Cohort).J Bone Miner Metab 2013;31:231-9.

16. Dominguez Cabrera C, Sosa Henríquez M, Traba ML, Alvarez Villafañe E, de la Piedra C. Biochemical markers of bone formation in the study of postmenopausal osteoporosis. Osteoporos Int 1998;8:147-51.

17. Rubert M, Martinez MJ, de la Piedra C. Normal values of the aminoterminal propeptide of type I collagen (PINP) and the isomer beta I collagen carboxyterminal telopeptide ( $\beta C T X)$ in serum of healthy premenopausal women of the Community of Madrid. Rev Osteoporos Metab Miner 2014;6:20-2.

18. Glover SJ, Garnero P, Naylor K, Rogers A, Eastell R. Establishing a reference range for bone turnover markers in young, healthy women. Bone 2008;42:623-30.

19. Bland JM, Altman DG. Statistical methods for assessing agreement between two methods of clinical measurement. Lancet 1986;1:307-10.

20. Bland, JM, Altman, DG. Comparing methods of measurement: why plotting difference against standard methods is misleading. Lancet 1995;346:1085-7.

21. Lin LI. A concordance correlation coefficient to evaluate reproducibility. Biometrics 1989;45:255-68.

22. Lin LI. A note on the concordance correlation coefficient. Biometrics 2000;56:324-5.

23. Available from: http://www.niwa.co.nz/node/104318/ concordance. Accessed 15 May, 2014.

24. Adami S, Bianchi G, Brandi ML, Giannini S, Ortolani S, DiMunno O, et al.; BONTURNO study group. Determinants of bone turnover markers in healthy premenopausal women. Calcif Tissue Int 2008;82:341-7.

25. Vasikaran S, Chubb P, Ebeling P, Jenkins N, Jones G, Kotowicz M, et al. Harmonized Australian reference intervals for serum PINP and CTX in adults. Clin Biochem Rev 2014;35:237-42.

26. Vasikaran S, Cooper C, Eastell R, Griesmacher A, Morris HA, Trenti T, et al. International Osteoporosis Foundation and International Federation of Clinical Chemistry and Laboratory Medicine position on bone marker standards in osteoporosis. Clin Chem Lab Med 2011;49:1271-4.

27. Vasikaran S, Eastell R, Bruyère 0 , Foldes AJ, Garnero $P$, Griesmacher A, et al. IOF-IFCC Bone Marker Standards Working Group. Markers of bone turnover for the prediction of fracture risk and monitoring of osteoporosis treatment: a need for international reference standards. Osteoporos Int 2011;22:391-420.

28. Krege JH, Lane NE, Harris JM, Miller PD. PINP as a biological response marker during teriparatide treatment for osteoporosis. Osteoporos Int 2014;25:2159-71. 